Artus de Bretagne. Roman en prose de la fin du XIII siècle et Arthur après Arthur. La matière arthurienne tardive en dehors du roman arthurien (1270-1530)

\title{
Hélène Bouget
}

\section{(2) OpenEdition}

Tournals

Édition électronique

URL : http://journals.openedition.org/abpo/3747

DOI : $10.4000 / a b p o .3747$

ISBN : 978-2-7535-7411-3

ISSN : 2108-6443

\section{Éditeur}

Presses universitaires de Rennes

Édition imprimée

Date de publication : 20 décembre 2017

Pagination : 200-203

ISBN : 978-2-7535-7409-0

ISSN : 0399-0826

\section{Référence électronique}

Hélène Bouget, « Artus de Bretagne. Roman en prose de la fin du xII" siècle et Arthur après Arthur. La matière arthurienne tardive en dehors du roman arthurien (1270-1530)", Annales de Bretagne et des Pays de l'Ouest [En ligne], 124-4 | 2017, mis en ligne le 20 décembre 2019, consulté le 23 septembre 2020. URL : http://journals.openedition.org/abpo/3747 ; DOI : https://doi.org/10.4000/abpo.3747

Ce document a été généré automatiquement le 23 septembre 2020.

(C) Presses universitaires de Rennes 


\section{Artus de Bretagne. Roman en prose de la fin $\mathrm{du}$ XIII ${ }^{e}$ siècle et Arthur après Arthur. La matière arthurienne tardive
en dehors du roman arthurien} (1270-1530)

Hélène Bouget

\section{RÉFÉRENCE}

FERLAMPIN-ACHER Christine, Artus de Bretagne. Roman en prose de la fin du XIII siècle, Édition critique du manuscrit BnF fr. 761 par, Paris, Champion, CFMA nº 180, 2017, 2 vol. (t. i : IX-CCXXXV + 518 p. ; t. ii : p. 519-871).

FERLAMPIN-ACHER Christine, (sous la direction de), Arthur après Arthur. La matière arthurienne tardive en dehors du roman arthurien (1270-1530), Rennes, Presses Universitaires de Rennes, 2017, 661 p.

Ces deux publications, éditées par Christine Ferlampin-Acher, sont issues de recherches concomitantes et complémentaires consacrées à la matière et au roman arthuriens tardifs, c'est-à-dire composés après 1270 et les grands cycles du Lancelot en prose, du Tristan en prose ou le roman de Guiron le courtois, à une époque où « le roman arthurien n'est plus aussi prisé que dans les années 1170-1250» (Arthur après Arthur, p. 7-8). Sur le plan narratif en effet, « le roi Arthur est mort [...], le Graal est remonté au ciel (p. 8). », mais si le roman arthurien semble s'épuiser, Arthur reste un référent culturel important, comme en témoigne l'ouverture du roman d'Artus de Bretagne dont l'action se situe explicitement "Apréz la mort le bon roy Artus, qui tant fu nobles roys et gentilz » Artus de Bretagne, t. I, p. 3, §1, 1-2. 
2 Contrairement à ce que le titre pourrait laisser entendre, le héros d'Artus de Bretagne n'est donc pas le roi Arthur, mais le fils de Jean, duc de Bretagne, et de la fille du comte de Lancastre en Angleterre. Ses aventures commencent en Petite-Bretagne, sur une terre marginale dans les romans arthuriens composés avant le milieu du xiIIe siècle. Ainsi, «Artus renouvellerait une matière épuisée en colonisant les marches spatiotemporelles de l'univers arthurien" t. I, p. CLXXIV. La narration est complexe, enchaînant rencontres amoureuses, conflits guerriers et merveilles féériques. Après avoir rejoué l'enfance sauvage de Perceval et les amours secrètes de Tristan et Iseut, le roman nous entraîne hors de la Bretagne armoricaine, tour à tour en Sorelois - une terre orientale -, au Danemark, à Corinthe, à Vienne, dans le royaume de France, comme dans des lieux imaginaires et merveilleux : la Porte Noire, l'île Perdue... Il convoque des personnages qui peuvent avoir un ancrage référentiel, comme le roi de Majorque, un comte de Montbéliard, de Nevers ou de Foix, et des personnages purement romanesques comme la fée Proserpine ou bien Florence, la fille du roi oriental Emenidus, qu'Artus finit par épouser avec l'aide d'un clerc aux pouvoirs surpuissants.

3 L'édition de C. Ferlampin-Acher offre pour la première fois au public l'opportunité de lire ce roman resté célèbre tout au long du Moyen Âge et qui a été imprimé de nombreuses fois jusqu'en 1628 (la bibliographie établie par l'éditrice donne utilement les références des 16 éditions répertoriées). Jusqu'à présent en effet, le lecteur n'avait à sa disposition que le fac-similé de l'édition de 1584 publié en 1996 par Nicole Cazauran et C. Ferlampin-Acher (Artus de Bretagne, fac-similé de l'édition de 1584, présentation par N. Cazauran et C. Ferlampin-Acher, Paris, Presses de l'École normale supérieure, 1996). L'introduction, qui livre sur plus de 200 pages une étude dense et synthétique de l'œuvre, permet de situer avec précision le contexte historique et culturel. Le roman a probablement été écrit en Bretagne, à un moment où la Bretagne se rapprochait de la France, entre 1296 et 1305. Sur la question cruciale de la datation, C. Ferlampin-Acher développe une argumentation très serrée fondée sur des données historiques, comme l'analogie entre les personnages du roman et le duc Jean II de Bretagne, père d'Arthur qui est « né en 1261, qui lui succède en 1305 et meurt en 1312 » (Artus de Bretagne, t. I, p. XXIII) ou bien encore la représentation des relations entre la France et l'Angleterre. C'est un roman d'avant les crises de succession du duché de Bretagne et la Guerre de Cent Ans (p. XcI), qui entretient, comme l'ensemble des romans en prose tardifs, « une relation spéculaire" (p.xxv) avec le réel et ancre sa fiction dans une onomastique réaliste (p. XXxIII). S'il n'est pas le reflet exact d'une situation politique, il peut se lire comme une "rêverie politique ", flattant un duc de Bretagne sensible à l'invention d'un passé mythique post-arthurien au duché.

4 Le manuscrit de base de l'édition (Paris, Bibliothèque nationale de France, français 761) est le plus ancien manuscrit conservé du roman et C. Ferlampin-Acher le date des années 1325-1350. La comparaison des différents manuscrits (14) révèle que la fin du roman est très mouvante : dans le manuscrit édité, le récit s'achève ainsi brutalement au cours du tournoi qui suit le mariage d'Artus, alors qu'ailleurs il se poursuit jusqu'à la mort du héros. De façon tout à fait originale mais aussi très logique, le classement des manuscrits proposé par C. Ferlampin-Acher se fonde sur les différentes fins répertoriées et permet de distinguer trois grandes versions du roman. Le texte édité appartient selon $\mathrm{C}$. Ferlampin-Acher à une première version, la plus courte, qui distingue d'ailleurs cinq fins possibles, mais qui n'est pas une réduction d'une deuxième 
version, plus longue. L'étude comparée des versions et des fins du roman permet d'aboutir à une conclusion importante dans le champ de la poétique des romans médiévaux: Artus de Bretagne se développe en effet par adjonction de continuations, comme les romans en vers et en prose de la première moitié du xiIIe siècle, à la différence des romans du xive et du xve siècle davantage clos sur eux-mêmes. Quant aux différentes versions, tantôt elles témoignent d'une "désarthurianisation du récit " (p. cI), tantôt elles remettent l'accent sur le lignage breton et varient selon le milieu où elles ont été copiées et lues.

5 Enfin, la partie de l'introduction consacrée à l'étude de la langue du manuscrit (p. cIXCLXXI) vient conforter les hypothèses émises dans l'analyse littéraire et l'étude contextuelle. L'état de la langue intermédiaire entre l'ancien et le moyen français accrédite l'idée d'une datation basse (entre le xIIIe et le xive siècle), bien que le maintien du système de flexion puisse témoigner «d'une volonté d'archaïsme [...] en adéquation avec un genre déclinant et nostalgique» (p.CLXX). Quelques traits dialectaux de l'Ouest corroborent l'idée que le texte a pu être écrit à la cour de Bretagne, mais d'une manière plus générale, la langue du manuscrit correspond aux usages littéraires parisiens où il a été copié.

6 L'ouvrage collectif Arthur après Arthur, issu des actes du séminaire du CETM de l'université Rennes 2 (2013-2015) et du projet LATE (littérature arthurienne tardive en Europe) initié par C. Ferlampin-Acher, ouvre la perspective au-delà du genre romanesque. Le volume contient 30 articles rassemblés dans une perspective synthétique comme en témoignent les introduction et conclusion générales très étoffées de C. Ferlampin-Acher. Malgré la diversité des approches et du corpus diversité d'ailleurs inhérente au projet - l'éditrice (également auteure de deux articles, l'un consacré aux épisodes arthuriens dans Laurin, Sone de Nansay et Aquilon de Bavière, p.137-155; l'autre à la «culture arthurienne» dans le Roman du Hem et le Roman de Guillaume d'Orange, p. 517-538), parvient, à travers une présentation précise des enjeux de l'ouvrage («Introduction», p. 7-21) et à l'établissement d'un bilan ouvert sur les recherches à mener ("Conclusion », p. 539-560), à produire un ouvrage d'une grande cohérence et à favoriser les échos et rappels entre les contributions.

7 Constatant la faible production arthurienne après 1270 et "l'obsolescence » des «valeurs chevaleresques prônées par la matière arthurienne [...] à la fin du Moyen Âge » (p. 8), C. Ferlampin-Acher propose d'observer les mentions et allusions à cette matière arthurienne dans d'autres genres littéraires. L'entreprise nécessite une mise au point théorique, rapide mais utile, sur des notions littéraires complexes qui seront convoquées dans la plupart des études, en particulier les notions de genre, de matière, de motif, d'intertextualité et de transfictionnalité. Elle évite par ailleurs deux écueils qui pouvaient a priori nuire à la démarche scientifique : s'arrêter à « la maigreur de la moisson» (p. 539) dans la collecte des attestations, et la difficulté de justifier d'une section temporelle (1270-1530) qui enjambe la périodisation traditionnelle entre Moyen Âge et Renaissance, et que C. Ferlampin-Acher considère comme « un troisième Moyen Âge, après les temps prélittéraires et les siècles centraux » (p. 21).

Le volume est organisé en trois parties. La première, «Le temps du roy Artus et des compaignons de la Table Ronde (Méliadus et Clériadice, § 1,1) : histoire(s) arthurienne(s) » montre la marginalisation du chronotope arthurien dans des romans, des chansons de geste, des chroniques où la matière arthurienne n'est souvent plus qu'un souvenir lointain, au mieux une couleur (dans Méliadus et Clériadice étudié par M. Szkilnik) ou 
un outil favorable à la création d'un « horizon arthurien » (dans les romans de Mélusine étudiés par J. Pavlevski-Malingre). Dans les chroniques, le personnel arthurien peut être davantage sollicité par nostalgie (chez Froissart étudié par P. Victorin) ou pour redessiner des événements historiques, comme dans les chroniques bretonnes du xve siècle étudiées par G. Péron et La Chronique d'Anjou qui évoque le personnage de Lancelot. Il me semble toutefois difficile d'admettre de façon aussi catégorique que l'auteur que le Lancelot-Graal, composé au xiIIe siècle, situe explicitement la légende de Lancelot en Anjou, les descriptions topographiques étant souvent topiques dans les romans arthuriens du xiIIe siècle, sans que l'on puisse aisément assimiler un toponyme actuel comme "la forêt de Beaufort-en-vallée " avec "le site du lac de Viviane " (p. 246). La deuxième partie, "Il n'y eut si petit qui a celle heure ne cuidast valoir Lancelot (Le livre du Cœur d'Amour Epris, XLV, L. 4) : modèles arthuriens ", met en évidence la valeur exemplaire, quoique parfois distanciée, des héros et textes arthuriens dans des textes aussi variés que Jean de Saintré (S. Lefèvre), les biographies chevaleresques (E. Gaucher-Rémond), les textes allégoriques (F. Pomel) ou, plus inattendus, les traités d'alchimie dans lesquels apparaissent régulièrement les noms de Merlin et d'Arthur. D. Kahn analyse « la vogue [...] des lectures alchimiques de romans arthuriens » (p. 376) à partir notamment de «la doctrine du sens caché» (p. 370) de Jacques Gohory. De tous les modèles, ceux de Chrétien de Troyes et du Chevalier au lion semblent avoir particulièrement retenu l'attention des auteurs: chez Guillaume de Machaut (J. Cerquiglini-Toulet), Guillaume de Lorris, Huon de Méry (F. Pomel), mais aussi dans Renart le Contrefait qui, comme le montre A. Barre, « contrefait » la poétique du Chevalier de la charrette, mais aussi, faut-il ajouter, reprend et réécrit en ouverture les premiers vers du Chevalier au Lion. La troisième partie : «Artus de Bretaigne [...] et maint autre dont je sui certaine que vous avez oÿ parler (Guillaume de Machaut, Le Voir Dit, VII) : une mode arthurienne? ", évalue la place de la matière arthurienne dans la culture commune et constate «la forte plasticité » (p. 381) de cette matière qui prend chez les Grands Rhétoriqueurs une couleur légendaire ( $\mathrm{E}$. Doudet) ou bien fonctionne au théâtre (V. Dominguez, R. Trachsler) "comme un réservoir d'allusions» (p. 409). Qu'elle soit dévaluée ou détournée dans les textes qui s'en font l'écho, la littérature arthurienne fait cependant toujours bien partie de la culture aristocratique, comme en témoignent les inventaires de bibliothèques (S. Fourcade), simplement parce que la noblesse peut encore s'identifier à l'univers arthurien, ou bien parce que cette littérature contribue à la construction identitaire d'une cour comme celle de Bourgogne (T. Van Hemelryck, D. Quéruel).

9 Il faut enfin saluer outre la bibliographie générale, la présence en annexe d'un relevé des attestations arthuriennes (p.561-611) qui reprend l'ensemble des références fournies dans les différentes contributions. 